\title{
Radiation Generation with an Existing Demonstrator of an Energy-Recovery Continuous-Wave Superconducting RF Accelerator
}

\author{
Ji-Gwang Hwang, * Michael Abo-Bakr, Aleksandr Matveenko and Georgios Kourkafas \\ Helmholtz-Zentrum Berlin (HZB), Albert-Einstein-Straße 15, Berlin 12489, Germany \\ Thorsten KAMPS ${ }^{\dagger}$ \\ Helmholtz-Zentrum Berlin (HZB), Albert-Einstein-Straße 15, Berlin 12489, Germany and \\ Humboldt-Universität zu Berlin Institut für Physik, Newton-Straße 15, Berlin 12489, Germany
}

(Received 8 January 2020; accepted 20 Febuary 2020)

\begin{abstract}
Over the past decades, many accelerator laboratories have put much effort into the development of compact energy-recovery linac (ERL) demonstrators to verify various physical and technical aspects of the generation, acceleration, transport and energy recovery of high brightness and high average current electron beams in a superconducting radio-frequency (SRF) linear accelerator. Beyond these goals, the ERL demonstrator also offers unique opportunities to study novel schemes for $\mathrm{THz}$ and X-ray radiation generation. In this paper, we discuss feasible options for schemes generating $\mathrm{THz}$ and X-ray radiation at low-energy continuous-wave (CW) SRF ERL demonstrators such as the bERLinPro accelerator.
\end{abstract}

Keywords: THz source, Energy recovery linac demonstrator, bERLinPro DOI: $10.3938 /$ jkps.77.337

\section{INTRODUCTION}

During the past two decades, the design and construction of energy recovery linear accelerators (ERLs), such as cERL in Japan [1], bERLinPro [2], S-DALINAC [3], and MESA [4] in Germany, ALICE in UK [5], PERLE in France [6], CBETA [7], ER@CEBAF [8] and CeC@RHIC in USA [9], and NovoFEL in Russia [10], have been carried out all over the world to demonstrate key technologies for a $\mathrm{GeV}$-scale energy-recovery linac (ERL)-based radiation source. Almost all of these accelerators utilize continuous-wave (CW) superconducting cavities to accelerate ultra-bright electron beams in 6-dimensional phase space at high repetition rates. This combination is substantial for obtaining high average and peak brightnesses as in lightsources based on storage rings, where the only task of accelerating cavities is to replenish losses arising from the emission of synchrotron radiation. Recently, the fundamental technology developments of a superconducting radio-frequency (SRF) high-power electron gun, high quantum efficiency photocathode and high-gradient accelerating cavities have been coming together gradually and the operational techniques for energy recovery have been proven with a beam current of 5-9 $\mathrm{mA}[11$, $12]$.

Here, we want to discuss science cases and applications

\footnotetext{
*E-mail: ji-gwang.hwang@helmholtz-berlin.de
}

${ }^{\dagger}$ E-mail: kamps@helmholtz-berlin.de beyond accelerator science research for bERLinPro [13], which is an ERL project and currently under construction at the Helmholtz-Zentrum Berlin (HZB) Institute in Germany. Various options have been discussed at the $63^{r d}$ ICFA Advanced Beam Dynamics Workshop on Energy Recovery Linacs 2019 (ERL'19) and at a satellite meeting [14]. One option was to use the low-energy ERL demonstrator as a hadron beam cooler for collider experiments. For this purpose, ampere-class fresh bunches are required to achieve sufficient cooling efficiency [15, 16]. Another proposal was to investigate capabilities for the generation of the wavelengths from Terahertz $(\mathrm{THz})$ to X-rays. ERL $\mathrm{THz}$ based research has gained significant attention over the past 20 years as sources and experiments have been developed in what was formerly a dark region of the electromagnetic spectrum [17]. Available space in the straight sections of the ERL provide an opportunity to install undulators or other special devices for future experiments to demonstrate the potential of ERLs for user applications. The high repetition-rate of the ERLs can easily achieve high average radiation power.

\section{BERLINPRO}

A sketch of the bERLinPro accelerator can be seen in Fig. 1. The bERLinPro injector, consisting of a photo- 


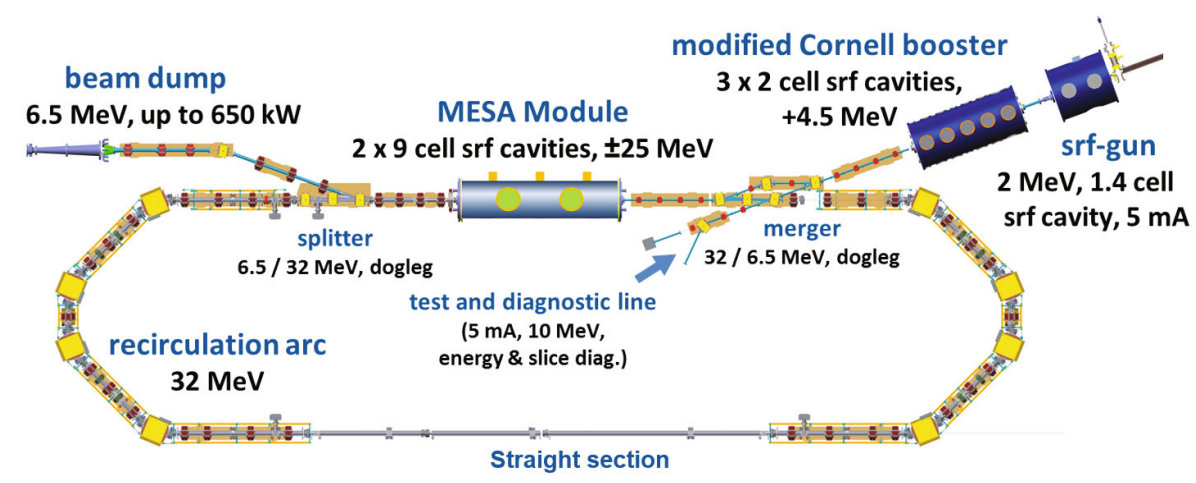

Fig. 1. bERLinPro - with a MESA module as the main linac.

Table 1. bERLinPro's main target parameters, initial project goals before descoping parenthesized.

\begin{tabular}{lc}
\hline \hline parameter & value \\
\hline maximum beam energy / MeV & $32(50)$ \\
maximum average current ${ }^{a} / \mathrm{mA}$ & $5(100)$ \\
RF freq. \& max. rep. rate / GHz & 1.3 \\
reference bunch charge / pC & 77 \\
normalized emittance / $\mu \mathrm{m} \mathrm{rad}$ & 1.0 \\
bunch length (standard mode) / ps & 2.0 \\
bunch length (short pulse mode ${ }^{b}$ / fs & 100 \\
maximum losses & $<10^{-5}$ \\
\hline \hline
\end{tabular}

${ }^{a}$ limited by the gun maximum coupler power or to lower values by beam break up (BBU)

$b$ at reduced bunch charge

cathode SRF gun (1.4 cell), followed by a booster cryomodule containing three SRF cavities (2 cells), generates a high brilliance beam with an energy of up to $6.5 \mathrm{MeV}$. The beam from the injector is merged into the linac section by using a dogleg chicane. This section can handle beams with two different energies with a bunch spacing of $385 \mathrm{ps}(2.6 \mathrm{GHz})$. Two beams, a lowenergy fresh and a high-energy to-be-recovered beam, pass through the main linac to be accelerated and decelerated, respectively. Through a racetrack magnetic lattice, including a long straight section where optional experimental equipments could be installed, the accelerated beam will be recirculated to demonstrate effective energy recovery. The decelerated beam is sent into the dump line at low beam energy. The beam in the recirculator straight section has a transverse size of a few hundreds of micrometers $\left(\varepsilon_{n, x y} \approx 1.0 \mu \mathrm{m} \cdot \mathrm{rad}, \beta \sim 10 \mathrm{~m}\right.$, $\left.\gamma=63 \rightarrow \sigma_{x y} \sim 400 \mu \mathrm{m}\right)$ at $77 \mathrm{pC}$ and a variable pulse length of $2 \mathrm{ps}$ in the standard operation mode. In a dedicated short-pulse mode at reduced charge, the bunches can be compressed down to 100 fs or even less. The project's basic set of parameters is listed in Table 1.

Due to schedule, resources and budget reasons, a major descoping of the project became necessary [18].
Thus, bERLinPro will be operated with an SRF gun that is expected to generate a maximum current of about $5 \mathrm{~mA}$, which is determined by the installed $\mathrm{RF}$ power couplers. Also, the bERLinPro main linac had to be canceled and will not be part of the project anymore. However, acceleration and energy recovery is still planned in bERLinProthrough a collaboration with the MESA project based at the Johannes GutenbergUniversitt Mainz. With the so-called MESA option [19], the test operation of one of the two MESA-project [20] main linacs will give researchers a chance to characterize it with electron beams, to accelerate the beam in bERLinPro to an energy of about $32 \mathrm{MeV}$, and to demonstrate energy recovery. The operation period will start in the middle of 2021 and includes all steps from commissioning and recirculation to energy recovery.

\section{THZ GENERATION IN BERLINPRO}

Although efficient laser-based $\mathrm{THz}$ sources have now enabled novel experiments on specific sample systems, only accelerator-based $\mathrm{THz}$ sources provide wide tunability together with high intensities and high repetition rates beyond $100 \mathrm{kHz}$ [21]. These advantages of accelerator-based sources will enable broad applications to the most interesting scientific problems in the field [17]. Particularly, the frequency range of 3 to $20 \mathrm{THz}$, where vibrational resonances and relaxations in condensed matter occur, is difficult to cover by using existing laser-based sources. In order to achieve high average and peak power at accelerator-based sources, we have to use coherent radiation, in which the radiation power is proportional to the square of the number of electrons. Coherent emission of radiation appears when the wavelength of the emitted radiation is significantly longer than the particle bunch length. The radiation spectrum can be represented as a sum of coherent and incoherent parts $[22,23]$ :

$$
P\left(\omega, \sigma_{z}\right)=p(\omega)\left(N_{e}+N_{e}\left(N_{e}-1\right) g^{2}\left(\sigma_{z}, \omega\right)\right),
$$




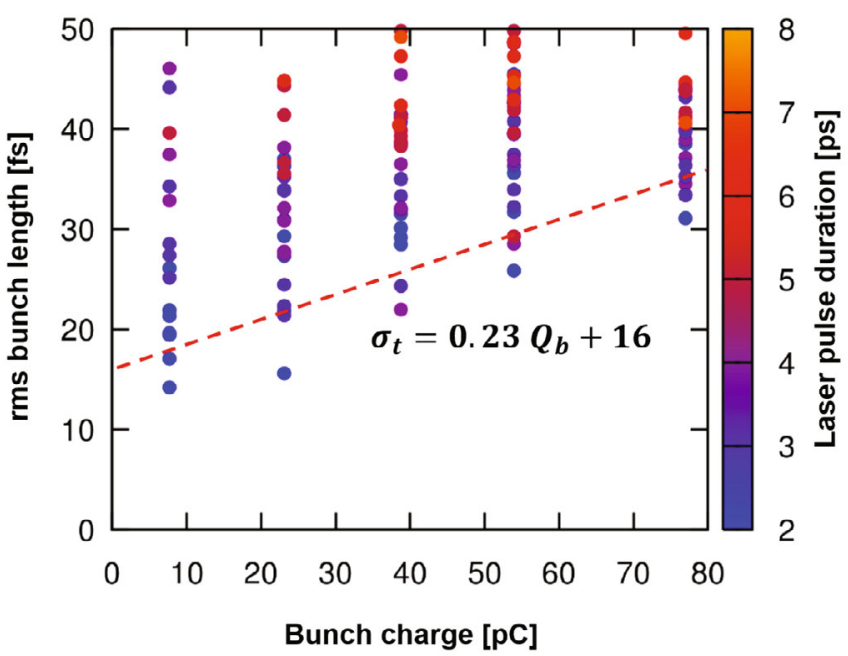

Fig. 2. Bunch length after compression as a function of bunch charge calculated for different parameter sets. The laser pulse length on the photocathode defines the color code. Details of the simulation are given in Ref. 28.

where $p(\omega)$ is the radiation power from one electron, $\omega=2 \pi f, N_{e}$ is the number of electrons, and $g\left(\sigma_{z}, \omega\right)$ is the form factor. The form factor is given by the modulus squared of the Fourier transform of the longitudinal charge density of the bunch. For a Gaussian particle distribution, $g^{2}\left(\sigma_{z}, \omega\right)=e^{-\frac{1}{2}\left(\frac{\sigma_{z} \omega}{c}\right)^{2}}$ is the form factor. The bunch form factor is equal to unity when the entire bunch acts as a single particle of enormous charge. For the generation of coherent radiation at frequencies of $1-$ $10 \mathrm{THz}$, we need to produce a bunch shorter than the form factor limit $\left(\sigma_{z}<<\sqrt{2} c / \omega\right)$. In bERLinPro, bunch lengths between $20 \sim 100$ fs can be achieved by using a two-stage compression scheme.

\section{Bunch Compression}

As we discussed above, one of the important questions for a $\mathrm{THz}$ source on the basis of the bERLinPro accelerator is, how short can the electron bunches be. The accelerator layout (see Fig. 1) and design of the magnetic optics for the accelerator are presented in Ref. 24. Bunch compression can be made in two compression stages by introducing a correlated linear energy chirp beforehand. The first possible stage includes the merger section in the injector. The necessary energy chirp is produced in the booster cavities. The limitations for the final bunch length after this stage are due to space-charge effects and the non-linearity of the RF field. The second stage uses a correlated energy spread produced by the main linac and the longitudinal dispersion of the recirculation arc. In this compression scheme, the final bunch length is limited by the coherent synchrotron radiation in the arc dipole magnets, as well as the $2^{n d}$ and higher order aberrations of the magnetic optics. Particle tracking simulations are necessary to estimate how short the bunches

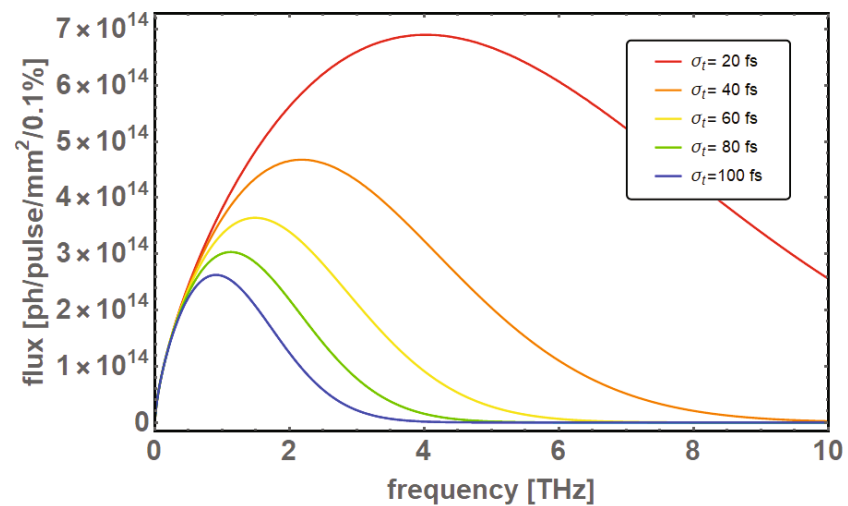

Fig. 3. Spectral distribution of the photon flux as a function of the bunch length with a dipole curvature of $0.78 \mathrm{~m}$, a bending angle of 45 degrees, an angular acceptance of the beamline of $10 \mathrm{mrad}$, a bunch charge of $20 \mathrm{pC}$, a normalized emittance of $1 \mathrm{~mm}-\mathrm{mrad}$ and an electron energy of $30 \mathrm{MeV}$.

can be and what is the optimal distribution of the compression between the two stages. The results depend on the bunch charge, laser pulse duration on the photocathode, and machine settings. ASTRA [25], CSRTrack [26], and Elegant [27] particle tracking codes were used for the simulations and optimizations of the machine settings for different bunch charges and laser pulse durations on the photocathode. A summary of the simulation results is shown in Fig. 2 [28].

The bunch length as a function of bunch charge is estimated from the fits to the average of a minimum of four cases of $\sigma_{t}(\mathrm{fs})=0.23 \times Q_{b}(\mathrm{pC})+16$, with a limit of $16 \mathrm{fs}$ for zero bunch charge. Due to the strong spacecharge forces at the shortest bunch lengths, a noticeable dilution, mainly of the horizontal emittance, cannot be avoided. However, the transverse emittance is still sufficiently small and well suited for the supposed $\mathrm{THz}-$ generation schemes.

2. Single-Cycle THz Generation by using Coherent Synchrotron Radiation

A bunch length of 20 fs, which corresponds to a form factor limit of $11.25 \mathrm{THz}$, can be achieved with a bunch charge of $17 \mathrm{pC}$, as extrapolated from the simulation results shown in Fig. 2. The dipole magnet installed in the recirculation arc is then a possible source of coherent synchrotron radiation (CSR) for intensive, wideband single-cycle $\mathrm{THz}$ radiation. This single-cycle short pulse allows studying fast dynamics, such as magnetic property switching in matter by electron paramagnetic resonance (EPR). The radiation power from one electron for synchrotron radiation (SR) is given by [29]

$$
p(\omega)=\frac{\sqrt{3} e^{2}}{4 \pi \epsilon_{0} c} \gamma \frac{\omega}{\omega_{c}} \int_{\omega / \omega_{c}}^{\infty} d x K_{5 / 3}(x),
$$

where $e$ is the charge of an electron, $\epsilon_{0}$ is the vacuum per- 
mittivity, $c$ is the speed of light, $\gamma$ is the Lorentz factor, $K_{n}(x)$ is a modified Bessel function of the second kind, and $\omega_{c}=3 c \gamma^{3} /(2 \rho)$ is the critical frequency, with $\rho$ being the curvature of the electron trajectory. From Eq. 2, the critical frequency of the SR from the arc dipole magnet is about $18 \mathrm{THz}$ for a dipole curvature of $0.78 \mathrm{~m}$ and an electron energy of $30 \mathrm{MeV}$. Thus, the wavelength of the radiation is mainly limited by the form factor. Due to the low electron energy, the SR has a relatively large divergence, $\Delta \phi=1 / \gamma=17 \mathrm{mrad}$. In order to confirm the achievable photon flux of the CSR from the dipole magnet, to calculate the spectral distribution as a function of the bunch length, we used the SPECTRA code [30] with an actual angular acceptance of a bERLinPro $\mathrm{THz}$ beamline and a bunch charge of $20 \mathrm{pC}$ and the results are shown in Fig. 3. With a bunch charge of $20 \mathrm{pC}$ and a length of $20 \mathrm{fs}$, the bERLinPro main dipole magnet in the recirculator can produce a photon flux of $6.7 \times 10^{14}$ photons/pulse $/ \mathrm{mm}^{2} / 0.1 \%$ B.W. up to a frequency of 11 $\mathrm{THz}$ with a bandwidth of $8.7 \mathrm{THz}$ at full width at half maximum (FWHM).

\section{High Pulse Power THz Generation by using} an Insertion Device

A strong demand exists for high peak power $\mathrm{THz}$ radiation for studying the nonlinear (NL) behaviors and the irreversible processes (phase transitions) in matter. The requirement for this is mainly a high electric field on a sample of $0.2 \mathrm{GV} / \mathrm{m}$ with a wide bandwidth of up to $30 \mathrm{THz}$ [17]. If a focus size diameter equal to the wavelength is assumed, the relation between the pulse energy $J_{\text {pulse }}$ and rms electric field $E_{r m s}$ is given by

$$
J_{\text {pulse }}=\frac{\pi \sigma_{\text {pulse }} c^{3}}{f^{2}} \epsilon_{0}\left|E_{r m s}\right|,
$$

where $\sigma_{\text {pulse }}$ is the pulse duration and $f$ is the carrier frequency. With a pulse duration of 1 ps ( $\sim 10$ cycles $)$, a electric field of $0.2 \mathrm{GV} / \mathrm{m}$ then corresponds to a pulse energies of $3 \mathrm{~mJ}$ at $0.1 \mathrm{THz}$ and $30 \mu \mathrm{J}$ at $1 \mathrm{THz}$. This pulse power can be obtained by installing an insertion device at the straight section of the recirculation arc, placed opposite to the linac section. If high-power SR pulses are to be generated in the $\mathrm{THz}$ region, an insertion device with a relatively long undulator period length of more than $18 \mathrm{~cm}$ is required. An in-house developed electromagnetic undulator (U180) [31], which is used for a metrology science application, can be applied to the bERLinPro accelerator. The undulator has an undulator period of $18 \mathrm{~cm}$, a number of periods of 12 , and a maximum K-value of 7.7. The pulse power is calculated using bERLinPro beam parameters. Figure 4 shows that a bunch charge of $160 \mathrm{pC}$ can achieve a pulse power of $30 \mu \mathrm{J}$ at $1 \mathrm{THz}$ and $3 \mu \mathrm{J}$ at $3 \mathrm{THz}$ with the bunch length of $60 \mathrm{fs}$. From the measurement result of the quantum efficiency of $5 \%$ (laser power of $25 \mathrm{~W}$ ) of the bERLinPro photocathode [32], the maximum bunch charge in

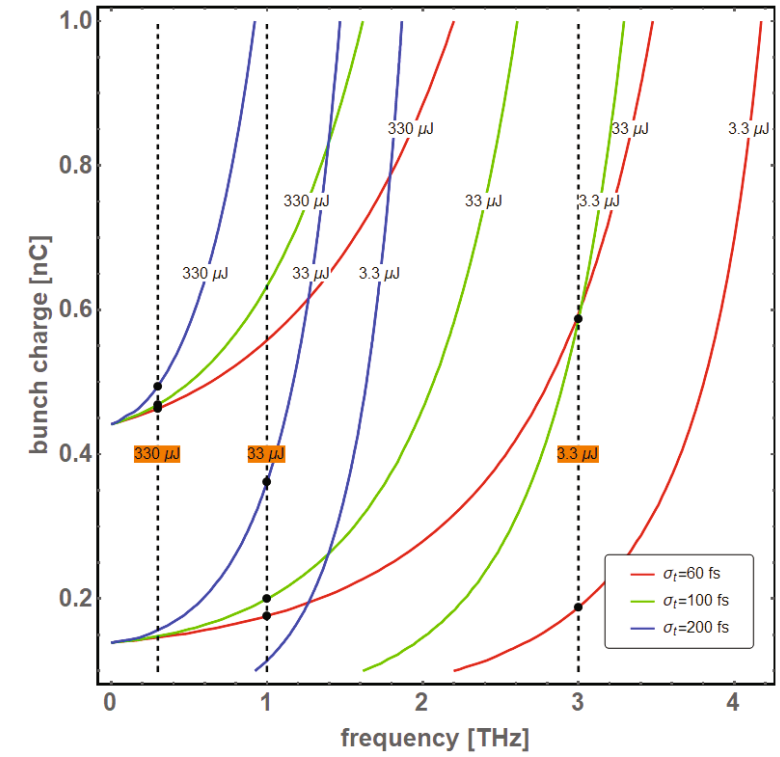

Fig. 4. Calculated SR pulse power with the specification of the U180 undulator as functions of frequency and bunch charge for bunch lengths of 60,100 , and $200 \mathrm{fs}$. The orange boxes show the pulse power required for an electric field of $0.2 \mathrm{GV} / \mathrm{m}$ at different frequencies. The dots represents the bunch charge required for an electric field of $0.2 \mathrm{GV} / \mathrm{m}$.

the bERLinPro is about $350 \mathrm{pC}$. Because the average current of the SRF electron gun is limited to $5 \mathrm{~mA}$, a bunch charge of $160 \mathrm{pC}$ with a relatively low repetition rate can be generated at bERLinPro.

\section{Compact $\mathrm{THz}$ Source by using Diffraction} Radiation

Recently, a novel scheme for $\mathrm{THz}$ generation has been proposed and proven by Honda [33]. The method uses diffraction radiation (DR) as source and an optical cavity for amplifying the radiation power significantly through a stimulation process. When a relativistic electron bunch passes a hole in the center of a metallic disk, radiation is generated by the diffraction of the electric field of the electron bunch at the surface of the metallic disk. In accordance with Ref. 34, the DR spectral-angular distribution generated by a single electron crossing a symmetric metallic-disk with a hole radius of $a$ can be represented by $[35]$

$$
\begin{aligned}
& p\left(\omega, \theta_{m}\right)= \frac{2 \alpha}{\pi^{2}} \int_{0}^{\theta_{m}} d \theta \frac{\theta^{3}}{\left(\theta^{2}+\gamma^{-2}\right)^{2}} \\
& \times\left[\frac{\omega b}{\gamma c} K_{1}\left(\frac{\omega b}{\gamma c}\right) J_{0}\left(\frac{\omega b}{c} \theta\right)\right. \\
&\left.\quad-\frac{\omega h}{\gamma c} K_{1}\left(\frac{\omega h}{\gamma c}\right) J_{0}\left(\frac{\omega h}{c} \theta\right)\right]^{2},
\end{aligned}
$$

where $\alpha$ is the fine structure constant, $\theta_{m}$ is the polar observation angle, $b$ is the outer radius of the disk, $h$ 


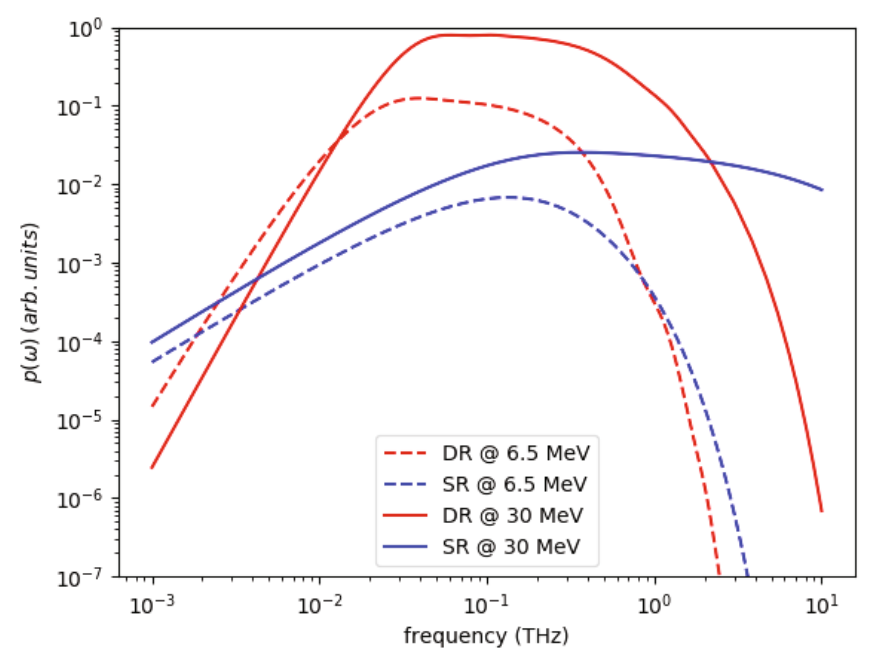

Fig. 5. Spectral power distribution of SR with an arc dipole magnet and a DR with a hole radius of $1.8 \mathrm{~mm}$ for electron energies of $6.5 \mathrm{MeV}$ (injector) and $30 \mathrm{MeV}$ (arc section).

is the Planck constant, and $J_{n}(x)$ is a Bessel function of the first kind. The DR power increases exponentially with decreasing hole radius $a$ when the hole radius is lager than $\gamma \lambda$. This $(a<<\gamma \lambda)$ is the criterion for generating intensive DR. Below this criterion, the DR power depends almost linearly on the the hole size. In the case of bERLinPro, due to the low energy, the criterion is a radius of $1.8 \mathrm{~mm}$ for $1 \mathrm{THz}$.

With Eqs. 2 and 4, the spectral power distribution of the $\mathrm{SR}$ and the DR with a hole radius of $1.8 \mathrm{~mm}$ for a single electron is calculated for electron energies of 6.5 and $30 \mathrm{MeV}$. Figure 5 shows that the DR photon flux is higher up to $2 \mathrm{THz}$ than the $\mathrm{SR}$ photon flux from the arc dipole magnet for an electron energy of $30 \mathrm{MeV}$. Particularly, the DR power can be amplified by inserting a small optical cavity. Due to the high repetition rate of the bERLinPro $(1.3 \mathrm{GHz})$, it can utilize a short optical resonator with a length of $11.5 \mathrm{~cm}$. With the combination of a high repetition rate and an optical cavity, the amplification of the $\mathrm{THz} \mathrm{DR}$ power by a factor of 1000 was experimentally confirmed [33]. Additionally, the output power can be enhanced by increasing the single-pass radiation power because the gain of the optical cavity is fundamentally determined by the cavity design. Here, we propose a new scheme for increasing the single-pass power by inserting a tapered corrugated structure in the middle of the optical cavity. Because a wavelength of $1 \mathrm{THz}$ corresponds to about $300 \mu \mathrm{m}$, a structure with about hundred surfaces can be installed within $3 \mathrm{~cm}$, which is about $1 / 4$ of the optical cavity length. Theoretically, the structure would increase the single-pass power by $10^{4}$ because the single-pass power is enhanced by the square of the number of metallic surfaces when the metallic surfaces are equally spaced with a distance equal to the target wavelength. However, the

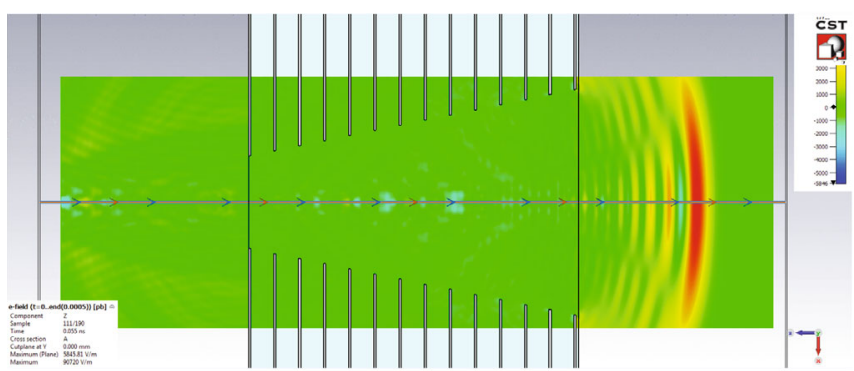

Fig. 6. Simulated electric-field distribution on the axis of electron travel in the DR from the tapered corrugated structure.

radiation power is exponentially reduced at the outward disk due to the large radius in the tapered structure, which is essentially required by the large angular divergence of the diffraction radiation. In order to confirm the efficiency of the structure, we need to simulate the DR from the tapered corrugate structure.

As shown in Fig. 6, the single-pass radiation has a multiple structure that indicates the enhancement of the radiation power explicitly. The backward radiation is, however, substantially trapped in the tapered structure. Further optimization of the internal structure is required for obtaining higher efficiency.

\section{X-RAY PULSE GENERATION AND ELECTRON PULSE FOR ULTRA-FAST SCATTERING}

The combination of $\mathrm{THz}$ radiation pulses and other $\mathrm{X}$ ray or electron pulses is required for performing pumpand-probe measurements that allow ultra-fast studies of the dynamics of atoms. For such applications, many scenarios for probe pulse generation are possible with bERLinPro. Depending on the particular user application, ultra-fast $\mathrm{THz}$, X-Ray or electron pulses can be supplied to an experimental station.

\section{X-ray Pulses from Compton Backscattering}

For sufficient signal-to-noise ratio at typical experiments and to allow for some transmission losses, at least 1000 to 5000 photons should be generated. Ultra-fast $\mathrm{X}$-ray pulses up to $20 \mathrm{-keV}$ photon energy could be generated by Compton backscattering of a $30-\mathrm{MeV}$ electron beam from the incoming photon pulse from a laser with an $800 \mathrm{~nm}$ wavelength laser. The length of the X-ray pulse should be shorter than 100 fs to allow dynamic studies of the electronic structure of matter. We used a linear model to estimate the photon flux and pulse length $[37,38]$ for the interaction of the photon pulses from a $800-n m$ wavelength, 10-mJ pulse energy and 100fs pulse length with electron pulses from the $\mathrm{NL} \mathrm{THz}$ 
scenario described above. This would result in $2 \times 10^{4}$ photons per shotfor a pulse length of $60 \mathrm{fs}$. Though the flux, if averaged over a $\mathrm{MHz}$ repetition rate, can be very high, the relatively large geometric emittance and the low beam energy lead to a large opening angle. Collimation would be required to allow efficient photon beam transport to the interaction region of the pump/probe experiment. Setting up the Compton interaction in a zig-zag geometry would lead to an increased photon flux and would even allow the Compton source to be operated in the two-color operation mode [39].

\section{Electron Pulses for Ultra-fast Scattering}

Another possibility to generate pulses to probe the atomic structure of matter is by electron scattering. Here, ultra-short relativistic electron beams at low bunch charge with $10^{4}-10^{6}$ electrons are focused on the experimental sample, and diffraction patterns are recorded or spectroscopy of the transmitted electron pulses is performed. The electrons are scattered by the Coulomb interaction with the binding electrons and with the protons inside the nucleus, so more information about the entire structure can be gained. For electron scattering experiments, the transverse coherence length of the electron pulses should be comparable to the domain size under investigation. The pulse length should also be shorter than 100 fs to allow dynamic studies of atomic motion.

We investigated an option to generate ultra-short electron pulses for scattering experiments in the beam diagnostic section behind the SRF gun and the SRF booster cavities. A highly coherent pulse is to be generated, a beam with a small spot size and low emittance needs to be launched in the SRF gun. This can be done by focusing the photocathode drive laser to extremely small spot sizes followed by rapid acceleration to keep emittance growth due to space charge effects under control. The ultra-short pulse length is generated by employing the SRF gun cavity and one SRF cavity in the SRF booster module for longitudinal phase space manipulation . With the so-called stretcher mode [40], the electron pulse at low charge is first stretched by acceleration at an offcrest phase in the SRF gun cavity and then longitudinally focused to a sub-fs pulse length at the interaction region with the SRF booster cavity [41]. In principle, researches should be able to interleave an ultra-low charge pulse with the electron pulses generated for the $\mathrm{THz}$ radiation process. The separation could be done with a fast RF kicker at the entrance of the merger beamline, where the high-charge pulses are fed on the axis of the main linac.

\section{CONCLUSION}

Various feasible schemes for generating high-power single- or multi-cycle $\mathrm{THz}$ radiation were studied with the beam parameters for bERLinPro. The main dipole magnets in the recirculator can produce high-power single-cycle $\mathrm{THz}$ radiation, which is usable for $\mathrm{THz}$ ERP applications. The installation of an additional U180 undulator at the recirculator straight section and a compact diffraction radiation source can produce high-pulsepower multi-cycle $\mathrm{THz}$ radiation suitable for the study of nonlinear behavior or irreversible processes. Especially, the short bunch mode for bunch lengths down to 20 fs offers a unique opportunity for generating strong coherent radiation over a wide spectral range from 3 to $10 \mathrm{THz}$, which is the most difficult to cover by existing sources. In addition, the bunch charge of $10 \mathrm{pC}$ to $200 \mathrm{pC}$ is capable of being used to study the material properties in the linear and nonlinear region. Furthermore, the X-ray pulses generated by using inverse Compton scattering of laser photons and ultrafast electron diffraction can be used to perform a fast dynamics study with a pump and probe method.

\section{ACKNOWLEDGMENTS}

Open Access funding enabled and organized by Projekt DEAL. This work was supported by the German Bundesministerium für Bildung und Forschung, Land Berlin and by grants from the Helmholtz Association.

Open Access: This article is licensed under a Creative Commons Attribution 4.0 International License, which permits use, sharing, adaptation, distribution and reproduction in any medium or format, as long as you give appropriate credit to the original author(s) and the source, provide a link to the Creative Commons licence, and indicate if changes were made. To view a copy of this licence, visit http://creativecommons. org/licenses/by/4.0.

\section{REFERENCES}

[1] S. Sakanaka et al., Nucl. Instrum. Methods Phys. Res. A 877, 197 (2018).

[2] M. Abo-Bakr et al., in Proc. 8th Int. Particle Accelerator Conf. (IPAC'17) (Copenhagen, Denmark, May 14-19, 2017), p. 855.

[3] A. Richter, in Proc. 5th European Particle Accelerator Conf. (EPAC'96) (Sitges, Spain, June 10-14, 1996), p. 110.

[4] T. Stengler et al., in Proc. 7th Int. Particle Accelerator Conf. (IPAC'16) (Busan, Korea, May 8-13, 2016), p. 2134 .

[5] F. Jackson et al., in Proc. 2nd Int. Particle Accelerator Conf. (IPAC'11) (San Sebastian, Spain, Sep. 4-9, 2011), p. 934.

[6] D. Angal-Kalinin et al., J. Phys. G 45, 065003 (2018). 
[7] D. Trbojevic et al., in Proc. 8th Int. Particle Accelerator Conf. (IPAC'17) (Copenhagen, Denmark, May 14-19, 2017), p. 1285.

[8] A. Bogacz et al., in Proc. 20th Particle Accelerator Conf. (PAC'03) (Portland, USA, May 12-16, 2003), p. 195.

[9] I. Ben-Zvi, in Proc. 10th European Particle Accelerator Conf. (EPAC'06) (Edinburgh, Scotland, June 26-30, 2006), p. 940.

[10] G. N. Kulipanov et al., IEEE Trans. Terahertz Sci. Technol. 5, 798 (2015).

[11] L. Merminga, in Proc. 22th Particle Accelerator Conf. (PAC'07) (New Mexico, USA, June 25-29, 2007), p. 22.

[12] P. Piot, D. R. Douglas and G. A. Krafft, Phys. Rev. ST Accel. Beams 6, 030702 (2003).

[13] M. Abo-Bakr et al., in Proc. 9th Int. Particle Accelerator Conf. (IPAC'18) (Vancouver, Canada, Apr.-May, 2018), p. 4127.

[14] T. Kamps et al., arXiv:1910.00881 [physics.acc-ph].

[15] S. Benson et al., presented at the 63rd Advanced ICFA Beam Dynamics Workshop on Energy Recovery Linacs (ERL'19) (Berlin, Germany, September 15-20, 2019).

[16] D. Kayran et al., presented at the 63rd Advanced ICFA Beam Dynamics Workshop on Energy Recovery Linacs (ERL'19), (Berlin, Germany, September 15-20, 2019).

[17] P. Zalden et al., "Technical note on Terahertz Sciene at European XFEL", April 2018, XFEL.EU TN-2018-00101.0 .

[18] A. Jankowiak et al., presented at the 63rd Advanced ICFA Beam Dynamics Workshop on Energy Recovery Linacs (ERL19) (Berlin, Germany, September 15-20 2019)

[19] B. C. Kuske et al., in Proc. 10th Int. Particle Accelerator Conf. (IPAC'19) (Melbourne, Australia, May 2019), p. 1449.

[20] F. Hug et al., presented at the 63rd Advanced ICFA Beam Dynamics Workshop on Energy Recovery Linacs (ERL'19) (Berlin, Germany, September 15-20, 2019), p. MOCOXBS05.

[21] B. Green et al., Sci. Rep. 6, 22256 (2016).

[22] E. Bründermann, H. Hübers and M. F. Kimmitt, Springer Series in Optical Sciences: Terahertz Techniques (Springer, 2012).
[23] J. Nodvick and D. Saxon, Phys. Rev. 96, 180 (1954).

[24] M. Abo-Bakr, B. C. Kuske and A. N. Matveenko, in Proc. 1st Int. Particle Accelerator Conf. (IPAC'10) (Kyoto, Japan, May 2010), p. 2135.

[25] K. Floettmann, "ASTRA, A Space Charge Tracking Algorithm", http://www.desy.de/ mpyflo/.

[26] M. Dohlus and T. Limberg, "CSRtrack", http://www. desy.de/xfel-beam/csrtrack/.

[27] M. Borland, "elegant: A Flexible SDDS-Compliant Code for Accelerator Simulation", Advanced Photon Source LS-287, September 2000.

[28] A. Ginter, Numerische Modellierung longitudinaler Dynamik bei bERLinPro, in German, MSc thesis, HU Berlin, 2016.

[29] J. D. Jackson, Classical Electrodynamics (3rd ed.) (Wiley, Chichester, 1999), p. 680.

[30] T. Tanaka and H. Kitamura, J. Synchrotron Radiat. 8, 221 (2001).

[31] R. Klein et al., J. Synchrotron Radiat. 5, 451 (1998).

[32] A. H. Schmeißer et al., Phys. Rev. Accel. Beams 21 , 113401 (2018).

[33] Y. Honda et al., Phys. Rev. Lett. 121, 184801 (2018).

[34] A. Potylitsyn, Nucl. Instrum. Methods Phys. Res. A 455 , 213 (2000).

[35] A. Aryshev et al., Nucl. Instrum. Methods Phys. Res. A 763, 424 (2014).

[36] CST-MWS website: https://www.cst.com/.

[37] S. K. Ride, E. Esarey and M. Baine, Phys. Rev. E 52 , 5425 (1995)

[38] W. J. Brown and F. V. Hartemann, in the 11th Advanced Accelerator Concepts Workshop, AIP Conf. Proc. No. 737 (AIP, New York, 2004), p. 839845.

[39] I. Drebot, in Proc. 9th Int. Particle Accelerator Conf. (IPAC'18) (Vancouver, Canada, May 19-24, 2019), p. 4196.

[40] B. Zeitler, K. Floettmann and F. Gruener, Phys. Rev. ST Accel. Beams 18, 120102 (2015).

[41] S. Koch, Numerical and analytical optimization of the bERLinPro SRF photoinjector and booster for short pulse operation, MSc thesis, HU Berlin, 2016. 